\title{
CLÁSICOS DEL CINE EN EDUCACIÓN INFANTIL
}

\author{
Antonia CASCALES MARTÍNEZ \\ C.P. Virgen de los Desamparados
}

Querer, entender, imaginar, sentir, etc. no son sino diversos modos del pensar que pertenecen al alma

Descartes.

Frente a la avalancha audiovisual, una de las mejores cosas a que puede aspirar el sistema educativo es ayudar a los alumnos a ir adquiriendo hábitos activos y críticos para que, en vez de caer en la hipnosis audiovisual, lleguen a ser espectadores activos. Y lo mejor empezar desde las bases del sistema, es decir, desde Educación Infantil.

Consideramos que desde la escuela es esencial prestar mayor atención al cine y su lenguaje, ya que la idea del mundo que tiene nuestros alumnos y alumnas es mucho más deudora de un imaginario del cine y la televisión que de lo que sus familias le han trasmitido.

En su famoso aforismo "el medio es el mensaje", McLuhan señalaba que cada medio de comunicación recrea la cultura y, en consecuencia, la transforma. En 1969, Enrico Fulchignoni, afirmó que hoy vivimos en la "Civilización de la Imagen".

El cine puede servir como documento de reflexión y debate, pero no lo podemos dejar de lado, puesto que se disfruta más de una película cuanto más aprendemos de ella, cuando nos descubre realidades que no conocíamos o cuando nos da nuevas perspectivas sobre temas más cercanos que creíamos conocer.

Partiendo de esta realidad, para enseñar a los niños de Educación Infantil a ver cine es necesario tener en cuenta los siguientes aspectos:

- En estas edades los niños no distinguen entre lo real y lo imaginario; pueden quedar "atrapados" por la realidad de la película sin saber salirse de ella.

- Para saber lo que un niño puede comprender de la película es necesario plantearse cuáles son las operaciones que es capaz de realizar y cuáles le exige el film.

- El sincretismo propio de esta etapa educativa choca con el esfuerzo de síntesis que exige la recepción de la película. 
- La falta de espíritu critico incapacita al niño para realizar un juicio personal y maduro de lo que está viendo.

- La comprensión de una película necesita una serie de actividades por parte del niño.

En la educación con respecto al cine atenderemos a dos aspectos:

- Enseñar a analizar al niño lo que ve, los mensajes que recibe, distinguiendo realidad de fantasía.

- Enseñar al niño a disfrutar del valor estético del cine.

- Conocer todo aquello que hace posible el cine: directores, actores, bandas sonoras...

Como es lógico, no son objetivos alcanzables durante la etapa de Educación Infantil, pero es el momento adecuado para empezar, puesto que se puede experimentar y motivar a nuestros alumnos con planteamientos concretos.

La propuesta didáctica que nosotros planteamos gira alrededor del cine clásico. Puede utilizarse en toda la etapa de Educación Infantil, puesto que contiene y sugiere unos contenidos que permiten un planteamiento interdisciplinar y globalizado.

Las películas, en nuestro caso Casablanca, Cleopatra y Lo que el viento se llevó, de las que se proyectan determinadas secuencias en Educación Infantil, sirven como motivación y toma de contacto para iniciar el proyecto de investigación.

Los pequeños proyectos de investigación, a pesar de que en su inicio se concibieron como una técnica didáctica, podemos definirlos como procedimientos de aprendizaje que permiten alcanzar unos objetivos a través de la puesta en práctica de una serie de acciones, interacción y recursos con motivo de resolver una situación o problema. Es un eje metodológico que permite garantizar un trabajo serio, riguroso y sistemático sobre cualquier objeto de estudio. Y se puede añadir que, más que una técnica didáctica, es una actitud ante la educación, concibiéndola como generadora de procesos que colaboran a que los niños lleguen a adquirir procedimientos para aprender de modo autónomo. Actualmente, esta definición se completa con la utilización de todo tipo de recursos digitales.

Si bien llevar el cine a las aulas de Educación Infantil es fácil, los que amamos el cine apostamos por que nuestros alumnos y alumnas entiendan que pasarlo bien con una 
película no está en absoluto reñido con el análisis y la reflexión. Pocos soportes unen tanto placer y aprendizaje como el cine o la literatura. Creemos firmemente que se disfruta mucho más de aquello que nos provoca aprendizaje, en el caso del cine, cuando una película nos descubre entornos lejanos que desconocíamos o cuando nos da nuevas perspectivas sobre asuntos más próximos que creíamos conocer.

La experiencia fílmica es personal, no se puede traspasar de unos a otros. Los alumnos de Educación Infantil, por supuesto, pueden llegar a disfrutar la película, pero debido a las características propias de su pensamiento precisarán de una carga de información complementaria superior a la de personas de más edad. Entendemos que el proceso de comunicación sólo acaba cuando somos capaces de sumar nuestra visión personal del mundo reconstruyendo desde nuestras experiencias el puzzle que nos ofrece el director.

Es evidente que sin reflexión se pierde el placer del cine. Cuando criticamos las películas que ven nuestros alumnos rara vez tenemos presente que, en esa escuela paralela que es el cine, buscamos aquello que necesitamos en función de nuestra realidad personal de ese momento. Unos buscan una educación sentimental, otros aventuras y una acción que no les da el entorno, otros descargar la adrenalina y hay quien solo busca el placer y la evasión. Si bien la idea evasión y placer es distinta a la de los adultos, y si lo que les mostramos como norma está alejado de sus intereses cotidianos difícilmente funcionará bien. Si pretendemos darle contenido didáctico al cine, debemos distinguir las diferentes sensibilidades de ellos y nosotros.

Para comprender cualquier lenguaje hemos de conocer sus códigos, reglas y vocabulario, y es evidente que lo manejaremos mejor cuanto mayor sea ese conocimiento. En cualquier caso, el cine ha ido formando su propio lenguaje, diferente del resto de las artes. Para comprender, degustar y gozar de una película hay que dominar las normas (cambiantes) del lenguaje cinematográfico e ir ampliando día a día nuestros conocimientos. Para comprender determinadas películas necesitaremos bastante nivel de conocimiento y experiencia.

El proyecto llevado a cabo sobre el cine clásico surgió en una actividad cotidiana, la asamblea. El interés por el tema fue inmediato y las maestras comprendimos que podíamos investigar sobre ello sin desmarcarnos del currículo propuesto para la etapa. Estábamos convencidas que se conseguirían los objetivos establecidos y se ampliarían los contenidos marcados. Este proyecto se ha desarrollado 
en el C.P. Virgen de los Desamparados, de Orihuela, con alumnos de segundo ciclo del tercer nivel de Educación Infantil.

El objetivo principal de nuestro proyecto es conocer los elementos que rodean el cine, despertando la curiosidad hacia el mismo. En la práctica, se trata de realizar pequeñas investigaciones para conocer los diferentes componentes de las películas seleccionadas, trasladando lo aprendido a aquellas películas que nos son más familiares.

\section{OBJETIVOS}

1. Introducción o habituación del cine clásico entre los alumnos.

2. Acercarse al lenguaje y los recursos del cine.

3. Conocer obras maestras alejadas de nuestra época: Casablanca, Cleopatra y Lo que el viento se llevó.

4. Tratar los eventos relacionados con el cine: Óscars y Goyas.

5. El cine como documento histórico.

\section{Desarrollo del proyecto}

Como ya se ha expuesto a lo largo del artículo, este proyecto gira en torno a tres películas de cine clásico: Casablanca, Cleopatra y Lo que el viento se llevó, analizando de cada una de ellas diferentes elementos.

En nuestro proceso de investigación se partió de los intereses de nuestros alumnos, partiendo de una canción de Barrio Sésamo, donde imitando a la película Casablanca cantan el abecedario. Nuestros alumnos se interesaron por la película, les hacía mucha gracia la expresión: "Tócala de nuevo, Sam”, pero desconocían quién era ese tal Sam. Aprovechamos ese interés para plantear el trabajo de investigación. Creímos que era la mejor ocasión para aprender cine, cine clásico concretamente.

\section{Primera etapa: Recopilación de la información}

El primer paso como en todo proyecto de investigación consiste en recopilar la información necesaria, así comenzamos pidiendo ayuda a nuestras familias con la siguiente frase:

$$
\begin{aligned}
& \text { El tiempo pasará } \\
& \text { mas tú recordarás... }
\end{aligned}
$$

Con este pareado, redactamos una nota informativa, que enviamos a las familias para solicitar información y documentación de las películas. Es obvio que los recursos 
con los que contamos en nuestras aulas son limitados y, en esta ocasión, del tema que vamos a trabajar no tenemos información alguna, para ello recurrimos a las familias de nuestros alumnos, cuya respuesta es excelente siempre.

Con la información recopilada, montamos en nuestra aula el rincón del cine clásico; además de las películas, contribuyeron con material impreso, muñecos, música, fotos y demás.

\section{Segunda etapa. Análisis de la información}

Lo primero que nos encontramos es que estas películas no son adecuadas para niños de cinco años, por ello no las pudimos ver enteras. El tema lo planteamos en la asamblea, y decidimos ver partes de cada una de ellas, permitiéndonos trabajar conceptos como: escenas, planos, secuencias, doblaje, actores principales, actores de reparto, banda sonora...

Partimos en todo momento de escenas y secuencias que, previamente, nosotras como profesionales habíamos revisado y estábamos convencidas de que nuestros alumnos podían entender.

Les llamó mucho la atención que había películas en blanco en negro, pensaban que la tele estaba rota. Esta circunstancia nos permitió explicar algo de los inicios del cine y de la fotografía.

\section{Tercera etapa. Análisis de Casablanca}

La primera película, como no podía ser de otra forma, fue Casablanca. Concretamente nos centramos en la secuencia donde se ven por primera vez en el bar los protagonistas. Ello nos dio la posibilidad de distinguir actores principales, Humphrey Bogart e Ingrid Bergman, de actores de reparto, Paul Henreid, Claude Rains, Conrad Veidt, entre otros.

Al centrarnos en una parte de la película, pudimos distinguir entre escenas y secuencias, creando nuestras propias secuencias a partir de las diferentes escenas que teníamos impresas sobre la película. Y luego creamos la secuencia de un día en clase.
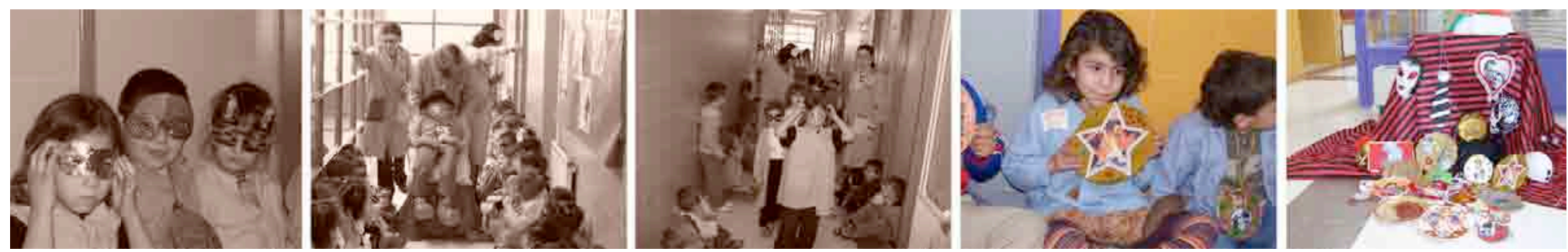
Y lo más importante, la banda sonora, "la música de las película" como la definió Noelia. Escuchamos la banda sonora de la película, y buscamos otras bandas sonoras de otras películas que conocíamos todos, como Cars, El rey león,... y aprovechamos para escribir la letra de la canción adaptada por Barrio Sésamo sobre Casablanca: "El abecedario".

\section{Cuarta etapa. Cleopatra}

La siguiente película fue Cleopatra, sobre esta película analizamos el argumento, el guión, el director, los decorados, los efectos especiales y los títulos de crédito. También estudiamos el argumento y el guión de la película, inventamos nuestros propios guiones:

Guión de la película: ¿DÓNDE ESTARÁ MI MADRE?

Autor: Joel Ballesteros González

Había una vez un niño llorando y le vio un policía.

Policía: ¿Por qué lloras?

Niño: Porque no encuentro a mi mamá.

Policía: Tranquilo, la buscaremos, ¿cómo es?

Niño: Es la más guapa del mundo.

Vieron a una mujer muy guapa.

Policía: ¿Es esa?

Niño: No, no es más guapa.

Vieron otra mujer muy guapa.

Policía: ¿Es aquella?

Niño: No, no es mucho más guapa.

Al fondo había una mujer muy fea con unas ropas muy raras.

Policía: ¿No será esa?

Niño: Sí, sí ¡es mi mamá!

Policía: Pero, pero, pero...

Niño: ¿A que es la más guapa del mundo?

Policía: Sí niño, tienes razón. No hay nada como una madre. 
Guión de la película: OTRO DÍA DE PLAYA

Autoras: Julia López Paredes y su madre

A Pedro no le gusta la playa, mientras que a Elena sí, pero no para ir dos horas, sino para ir todo el día, desde las ocho de la mañana hasta las diez de la noche.

Julia: Papá, papá, levanta, ya está todo preparado.

Pedro: Pero, ¿es que no entendéis que no me gusta la playa?

Elena: Ánimo, hoy será un día diferente.

Pedro: Sí, diferente, como cada vez que me pongo a pescar y no pesco nada.

Allá va Pedro resignándose, cargando con todo lo más pesado, el toldo, las hamacas, la nevera, la mesa, etc.

Julia: ¡Qué bien, ya estamos en la playa!

Elena: Julia, no te acerques tú sola al agua. Papá y yo vamos a montar el chiringuito.

Julia: No mamá, voy a construir un castillo.

Una vez todo montado se dieron un baño.

Pedro: Voy a pescar, tengo que traer el pescado para comer este mediodía.

Julia y su mamá se echaron una gran carcajada.

Julia: No te preocupes papá, mamá ya ha traído la comida hecha.

Pedro: ¿Qué graciosas?

Así pasaron la mañana...

Continuará

Al estudiar los decorados y vestuario decidimos vestirnos como la protagonista de la película y aprendimos cosas de su época, cuidando hasta el mínimo detalle.
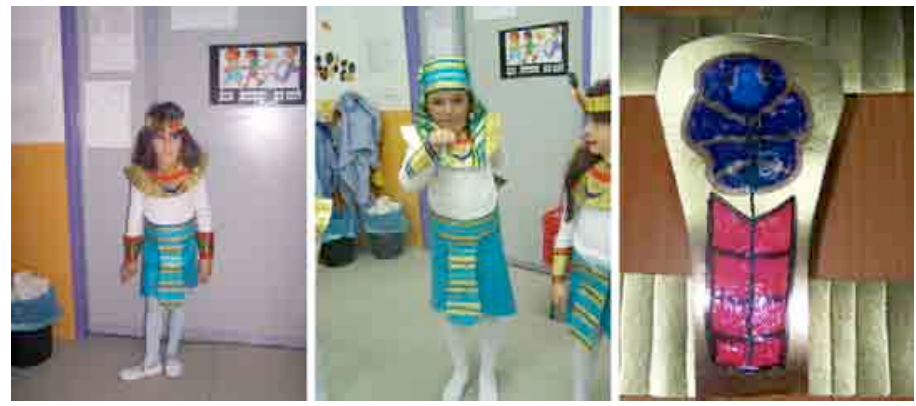

\section{Quinta etapa. Lo que el viento se llevó}

Finalmente, nos centramos en Lo que el viento se llevó, de ella destacamos el vestuario, el género, la cámara y por supuesto los premios que recibió, seis Óscars.

Con esta película recordamos todo cuanto habíamos aprendido, y nos centramos especialmente en el reconocimiento que se le otorga a un trabajo bien hecho, los 
premios que a todos nos gustan. En este caso los Goya en España y los Óscars en Estados Unidos.

Además de estudiar los que se llevó este film, aprovechamos para ver los que se habían concedido este año y fue una grata sorpresa saber que a películas que tanto nos gustan como Piratas del Caribe y Happy Feet les habían concedido un Óscar.

\section{Sexta etapa. Hacemos cine}

Para concluir este inmenso e intenso trabajo hicimos nuestra propia película, basada en la vida de la criada de Lo que el viento se llevó. Teniendo presente todo lo que habíamos estudiado: guión, actores, escenario, vestuario, escenas, secuencias.... Incluimos algo nuevo que nos gustó mucho, las tomas falsas.
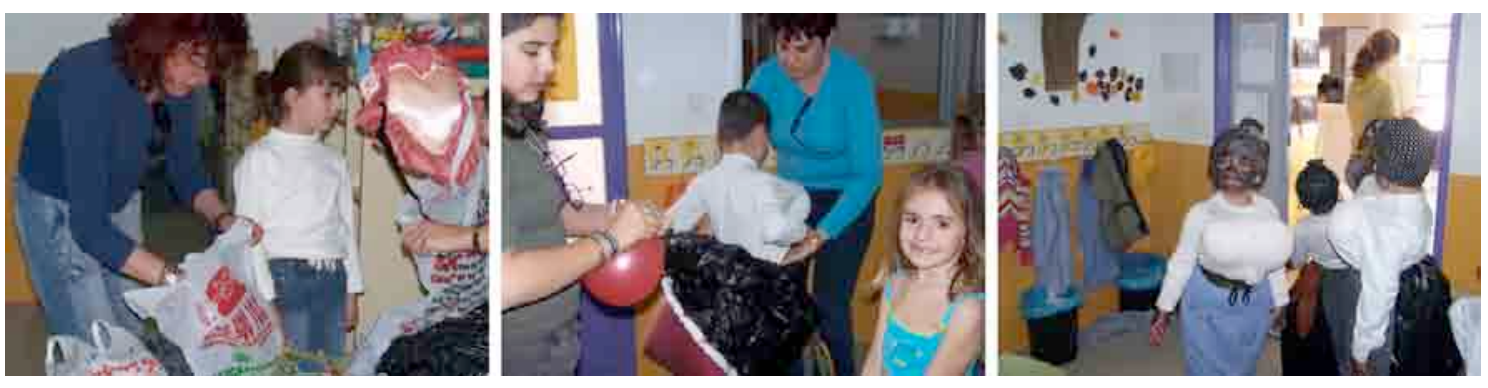

Somos conscientes de que se debe respetar el proceso natural del aprendizaje, implicando a los niños en la planificación y elaboración de una tarea común. Ello supone crear una dinámica en el aula, globalizada e individualizada que favorezca la cooperación, la tolerancia y el respeto entre todos. No podemos olvidar que debemos fomentar en el niño tanto la lógica, la deducción y la memorización como la fantasía; que nos llevará al empleo de procesos inductivos, imaginativos y creativos. Nuestros proyectos siguen un proceso de creación a través del método científico, lo que supone la materialización de algo concreto, salidas, talleres, rincones, transformaciones... en este caso nuestra primera película.

Estamos convencidas de que esta forma de trabajar ha calado en toda la comunidad educativa. En primer lugar, el equipo docente ha planteado el cine desde otra perspectiva a la habitual y para ello ha buscado métodos, técnicas y estrategias para trasmitirlo a los alumnos. Las familias han sido una parte sustancial del éxito de este proyecto. Si bien al principio les resultó extraño y llamativo, con el desarrollo del 
proyecto se han ido implicando y han aprendido a disfrutar de este tipo de cine. Finalmente, los alumnos han disfrutado conociendo todo lo que conlleva una película. 


\section{BIBLIOGRAFÍA:}

ROMAGUERA, Joaquim y otros, El cine en la escuela. Elementos para una didáctica, Barcelona, Gustavo Gili, 1989.

FERRÉS, Joan, Vídeo y educación, Barcelona, Paidós, 1992.

MASTERMAN, Len, La enseñanza de los medios de comunicación, Madrid, Ed. de la Torre, 1993.

COLL, Mercé y otras, (colectivo Drac Mágic) Cien años de cine. Guía para el uso del cine en la escuela, Monográfico de Cuadernos de Pedagogía, no 242 (diciembre, 1995).

FERNÁNDEZ, Carlos, Iniciación al lenguaje del cine, Madrid, Ministerio de Cultura, 1979.

MCLUHAN, Marshall, El medio es el mensaje. Barcelona, Paidós, 1987.

MARTIN, Marcel, El lenguaje del cine, Barcelona, Gedisa, 1990.

CHALLIER, Marion y JEUNET, Lou, Érase una vez el cine, Madrid, SM, 1994. 